\title{
A Spacecraft Attitude Tracking Control Method based on Disturbance Estimation
}

\author{
Guang Sun ${ }^{\mathrm{a}, *}$, Chunyang Zhang ${ }^{\mathrm{b}}$, and Yongyuan $\mathrm{Li}^{\mathrm{c}}$ \\ Research and Development Centre, China Academy of Launch Vehicle Technology, Beijing, China \\ asunlight17330@163.com, bspringsunjyjlvzcy@126.com, 'lyy6912@yeah.net \\ *Corresponding author
}

Keywords: Disturbance Estimation, Spacecraft Attitude Tracking, Control

\begin{abstract}
In this paper, a control method is proposed for spacecraft with unknown disturbances. For attitude subsystem, an adaptive estimator is designed to deal with the unknown disturbances. For angular velocity subsystem, the actual control input is designed. If the designed control law is added to angular velocity subsystem, a nonlinear closed-loop system of angular velocity error is arrived. The convergence of closed-loop system is proved by input-output stability theory. The derivative of virtual control and unknown disturbances in the actual control are also estimated using adaptive estimator. It can be proved that, with the proposed controller, the attitude can converge to a small-neighbourhood of the designed attitude. Theoretical results are illustrated by numerical simulation.
\end{abstract}

\section{Introduction}

To improve the anti-disturbance performance of control systems, disturbance-observer-based control strategies were designed in recent years. In [1], a high-order sliding mode observer based time-varying sliding mode control strategy is presented to address the robust attitude control problem. In [2], a disturbance observer-based exponential time-varying sliding mode attitude controller was designed to achieve precise attitude control when the matched parametric uncertainties and external disturbances occurred. Based on adaptive disturbance observer, an output feedback controller is employed to provide a solution to the robust tracking control problem of an electrically driven free-floating space manipulator with internal parameter uncertainties and external disturbances in [3]. In [4], a direct feedback linearization control method with nonlinear disturbance observer was proposed to achieve excellent control performance for permanent magnet synchronous motor. In [5], a nonlinear disturbance observer based command filtered backstepping control method is proposed for nonlinear missile system in face of multiple disturbances. In [6], to solve the control problem of a general hyper-sonic vehicle, a robust backstepping control strategy based on the disturbance- observer is proposed to estimate and compensate for disturbances by using the super-twisting algorithm; finite time convergence is guaranteed by the proposed control. In [7], non-singular terminal sliding mode control and finite-time disturbance observer were introduced to design the composite guidance law for the terminal guidance of missile to intercept maneuvering targets; disturbance rejection is guaranteed, and chattering phenomenon is alleviated.

Used aforementioned methods for reference, a new control method with estimate and offset about mismatch disturbance is proposed for a MIMO spacecraft subject to external disturbances in the paper. In control design, the mismatched disturbance is fully considered. Adaptive estimator and controller are designed for attitude subsystem and angular velocity subsystem, respectively. For angular velocity subsystem, the actual control input is designed. If the designed control law is added to angular velocity subsystem, a nonlinear closed-loop system of angular velocity error is arrived. The convergence of closed-loop system is proved by input-output stability theory. The derivative of virtual control and unknown disturbances in the actual control are also estimated and compensated using adaptive estimator. It can be proved that, with the proposed controller, tracking error of the attitude converges to a bounded small-neighborhood of the reference attitude. 


\section{Spacecraft Model for Attitude Control}

During the attitude control of spacecraft, it is susceptible to the orbital motion, measuring noise and other factors. For high-precision attitude control problems, these interfering factors can not be ignored. In the paper, attitude equation is described as following:

$$
\dot{\boldsymbol{q}}=\boldsymbol{B}(\boldsymbol{q}) \boldsymbol{\omega}+\boldsymbol{f}
$$

Where $\boldsymbol{B}$ is a reversible attitude rotation matrix when spacecraft attitude $\boldsymbol{q}$ change in a certain set (it is researched only that the case of $\boldsymbol{B}$ is reversible in the paper), $\boldsymbol{\omega}$ is attitude angular velocity, $f$ is disturbance of impacting on attitude system. The dynamic equation of spacecraft can be written by:

$$
J \dot{\omega}+\omega^{\times} J \omega=u+d
$$

Where $\boldsymbol{J}$ is the moment of inertia, $\boldsymbol{\omega}^{\times}$denotes the skew-symmetric cross product matrix corresponding to $\boldsymbol{\omega}, \boldsymbol{u}, \boldsymbol{d}$ are control vector and external disturbance, respectively.

\section{Attitude Tracking Control}

The objective of this paper is design a controller for system described by (1) and (2), such that the spacecraft attitude $\boldsymbol{q}(t)$ follows the desired attitude $\boldsymbol{q}_{r}(t)$ as closely as possible, that is

$$
\lim _{t \rightarrow \infty}\left(\boldsymbol{q}(t)-\boldsymbol{q}_{r}(t)\right)=0
$$

For spacecraft model (1) and (2), the strategy of attitude control is based on the following concerns: 1) a virtual predictive control is introduced to attitude subsystem, where the uncertainties are estimated and compensated by using adaptive estimator; 2) the actual control input is designed by constructing a nonlinear closed-loop system of angular velocity error, and adaptive estimator is planned again.

In this paper, we suppose and satisfy following conditions:

1) $\boldsymbol{d}$ and $\boldsymbol{q}_{r}(t)$ are bounded; 2) $\boldsymbol{q}_{r}(t)$ and its derivatives are bounded.

\subsection{Control Design for Attitude Subsystem}

Define attitude tracking error and angular velocity error:

$$
\begin{gathered}
\tilde{\boldsymbol{q}}(t)=\boldsymbol{q}_{r}(t)-\boldsymbol{q}(t) \\
\tilde{\boldsymbol{\omega}}(t)=\boldsymbol{\omega}_{d}(t)-\boldsymbol{\omega}(t)
\end{gathered}
$$

Where $\omega_{d}(t)$ is a virtual control input to be designed and $\boldsymbol{q}_{r}(t)$ is desired attitude trajectory.

For attitude subsystem, choose virtual control law as follows:

$$
\boldsymbol{\omega}_{d}(t)=\boldsymbol{B}^{-1}\left(\boldsymbol{K}_{d} \tilde{\boldsymbol{q}}+\dot{\boldsymbol{q}}_{r}+\boldsymbol{B} \tilde{\boldsymbol{\omega}}(t)-\boldsymbol{f}\right)
$$

Where $\boldsymbol{K}_{d}$ is a positive diagonal matrix. Substituting virtual control law (6) into spacecraft attitude motion equation (1) yields that

$$
\dot{\tilde{\boldsymbol{q}}}(t)+\boldsymbol{K}_{d} \tilde{\boldsymbol{q}}(t)=0
$$

Hence, (7) implies that $\lim _{t \rightarrow \infty} \tilde{\boldsymbol{q}}(t)=0$. It indicates that the tracking error of the closed-loop system is globally asymptotically stable.

The virtual control (6) is unable to implement, since there are unknown term $\boldsymbol{f}$. To compensate for the influence of the unknown terms in (6) meanwhile maintain the independence of control every subsystem, a feasible way is design a new control law to approximate the ideal control law (6). Let $\boldsymbol{T}_{n}=\boldsymbol{B} \tilde{\boldsymbol{\omega}}(t)-\boldsymbol{f}$, and suppose that $\left\|\boldsymbol{T}_{n}\right\| \leq \delta_{1}$, where $\delta_{1}>0$ is an unknown and bounded constant. 
Defined $\tilde{\delta}_{1}=\hat{\delta}_{1}-\delta_{1}$, which is estimate error of $\delta_{1}$, and $\hat{\delta}_{1}$ is estimate value of $\delta_{1}$. The control law can be redesigned as:

$$
\begin{gathered}
\boldsymbol{\omega}_{d}(t)=\boldsymbol{B}^{-1}\left(\boldsymbol{K}_{d} \tilde{\boldsymbol{q}}+\dot{\boldsymbol{q}}_{r}+\frac{c_{1} \hat{\delta}_{1}}{\|\tilde{\boldsymbol{q}}\|+b_{1}} \tilde{\boldsymbol{q}}\right) \\
\dot{\hat{\delta}}_{1}=\frac{a_{1} c_{1}}{\|\tilde{\boldsymbol{q}}\|+b_{1}} \tilde{\boldsymbol{q}}^{T} \tilde{\boldsymbol{q}}
\end{gathered}
$$

Where $a_{1}, b_{1}>0, c_{1}>1$ are constants to be designed. Define Lyapunov function $V_{1}=1 / 2 \tilde{\boldsymbol{q}}^{\mathrm{T}} \tilde{\boldsymbol{q}}+1 /\left(2 a_{1}\right) \tilde{\delta}_{1}^{2}$, Differentiating $V_{1}$ on time yields that

$$
\dot{V}_{1} \leq-\tilde{\boldsymbol{q}}^{\mathrm{T}} \boldsymbol{K}_{d} \tilde{\boldsymbol{q}}+\|\tilde{\boldsymbol{q}}\| \delta_{1}-\frac{c_{1} \delta_{1}}{\|\tilde{\boldsymbol{q}}\|+b_{1}} \tilde{\boldsymbol{q}}^{T} \tilde{\boldsymbol{q}} .
$$

\subsection{Control Design for Angular Velocity Subsystem}

For angular velocity subsystem, the control law is designed as follows:

$$
\boldsymbol{u}^{*}(t)=\boldsymbol{K}_{p} \boldsymbol{J}\left(\boldsymbol{\omega}_{d}-\boldsymbol{\omega}\right)+\boldsymbol{J} \dot{\boldsymbol{\omega}}_{d}+\boldsymbol{\omega}^{\times} \boldsymbol{J} \boldsymbol{\omega}_{d}-\boldsymbol{d}
$$

Where $\boldsymbol{K}_{p}$ is a positive diagonal matrix. Substituting control law (11) into spacecraft attitude dynamics equation (2) yields that the closed-loop system equation is

$$
\boldsymbol{J}\left(\dot{\boldsymbol{\omega}}_{d}-\dot{\boldsymbol{\omega}}\right)+\boldsymbol{\omega}^{\times} \boldsymbol{J}\left(\boldsymbol{\omega}_{d}-\boldsymbol{\omega}\right)+\boldsymbol{K}_{p} \boldsymbol{J}\left(\boldsymbol{\omega}_{d}-\boldsymbol{\omega}\right)=0
$$

That is different from the classical scheme of feedback control. The close-loop system equation derived from the designed control law is a nonlinear differential equation. A compact format of above-mentioned equation can be rewritten as

$$
\boldsymbol{J} \dot{\tilde{\boldsymbol{\omega}}}-\boldsymbol{\omega}^{\times} \boldsymbol{J} \tilde{\boldsymbol{\omega}}+\boldsymbol{K}_{p} \boldsymbol{J} \tilde{\boldsymbol{\omega}}=0
$$

Choose pseudo-Lyapunov function $V_{2}=1 / 2 \tilde{\boldsymbol{\omega}}^{\mathrm{T}} \boldsymbol{J}^{\mathrm{T}} \boldsymbol{J} \tilde{\boldsymbol{\omega}}$, Differentiating $V_{2}$ on time yields that

$$
\dot{V}_{2}=-\tilde{\boldsymbol{\omega}}^{\mathrm{T}} \boldsymbol{J}^{\mathrm{T}} \boldsymbol{K}_{p} \boldsymbol{J} \tilde{\boldsymbol{\omega}} \leq 0
$$

This means the trajectory of close-loop system $V_{2}(t)$ is monotonously and bounded, and $\forall t \geq t_{0} \geq 0$, one can get

$$
0 \leq V_{2}(\infty) \leq V_{2}(t) \leq V_{2}\left(t_{0}\right)<\infty
$$

Based on above inequality and definition of $V_{2}(t)$, one arrive

$$
0 \leq \frac{1}{2} \lambda_{\min }\left(\boldsymbol{J}^{\mathrm{T}} \boldsymbol{K}_{p} \boldsymbol{J}\right)\|\tilde{\boldsymbol{\omega}}\|^{2} \leq \frac{1}{2} \tilde{\boldsymbol{\omega}}^{\mathrm{T}} \boldsymbol{J}^{\mathrm{T}} \boldsymbol{K}_{p} \boldsymbol{J} \tilde{\boldsymbol{\omega}}<\infty
$$

Therefore, one has $\tilde{\boldsymbol{\omega}} \in L^{\infty}$. From equation (14), one can find

$$
\dot{V}_{2}(t)=-\tilde{\boldsymbol{\omega}}^{\mathrm{T}} \boldsymbol{J}^{\mathrm{T}} \boldsymbol{K}_{p} \boldsymbol{J} \tilde{\boldsymbol{\omega}} \leq-\lambda_{\min }\left(\boldsymbol{J}^{\mathrm{T}} \boldsymbol{K}_{p} \boldsymbol{J}\right)\|\tilde{\boldsymbol{\omega}}\|^{2}
$$

Integrating above inequality (17), one obtains

$$
\int_{t_{0}}^{\infty}\|\tilde{\boldsymbol{\omega}}\|^{2} \mathrm{~d} t \leq-\frac{1}{\lambda_{\min }\left(\boldsymbol{J}^{\mathrm{T}} \boldsymbol{K}_{p} \boldsymbol{J}\right)} \int_{t_{0}}^{\infty} \dot{V}_{2}(t) \mathrm{d} t=\frac{1}{\lambda_{\min }\left(\boldsymbol{J}^{\mathrm{T}} \boldsymbol{K}_{p} \boldsymbol{J}\right)}\left(V_{2}\left(t_{0}\right)-V_{2}(\infty)\right) \leq \frac{1}{\lambda_{\min }\left(\boldsymbol{J}^{\mathrm{T}} \boldsymbol{K}_{p} \boldsymbol{J}\right)} V_{2}\left(t_{0}\right)<\infty
$$

So that $\tilde{\boldsymbol{\omega}} \in L^{2}$.

According to the suppose with $\boldsymbol{q}_{r}(t)$ and the conclusion $\tilde{\boldsymbol{q}}(t) \in L^{\infty}$, one can get from the equation (8). Under the condition on the suppose of $\boldsymbol{d}$ and $\boldsymbol{q}_{r}(t)$, the control law satisfies: 


$$
\boldsymbol{u}^{*}(t)=\boldsymbol{K}_{p} \boldsymbol{J}\left(\boldsymbol{\omega}_{d}-\boldsymbol{\omega}\right)+\boldsymbol{J} \dot{\boldsymbol{\omega}}_{d}+\boldsymbol{\omega}^{\times} \boldsymbol{J} \boldsymbol{\omega}_{d}-\boldsymbol{d} \in L^{\infty}
$$

From the dynamic equation of spacecraft, one has

$$
\dot{\boldsymbol{\omega}}=\boldsymbol{J}^{-1}\left(\boldsymbol{\omega}^{\times} \boldsymbol{J} \boldsymbol{\omega}+\boldsymbol{u}+\boldsymbol{d}\right) \in L^{\infty}
$$

So that $\dot{\tilde{\boldsymbol{\omega}}} \in L^{\infty}$.

Based on the above analyze, $\tilde{\boldsymbol{\omega}} \in L^{2}$ and $\dot{\tilde{\boldsymbol{\omega}}} \in L^{\infty}$ is valid, and then $\lim _{t \rightarrow \infty} \tilde{\boldsymbol{\omega}}(t)=0$ is reasonable.

One can see from (11) that the term $\dot{\boldsymbol{\omega}}_{d}$ in the control law would greatly increase the computational burden, and the unknown terms $\boldsymbol{d}$ make the controller impracticability. In the section, the virtual control derivative $\dot{\boldsymbol{\omega}}_{d}$ is regarded as uncertainty to avoid the huge computational burden. Let $\boldsymbol{T}_{m}=\boldsymbol{J} \dot{\boldsymbol{\omega}}_{d}-\boldsymbol{d}$, and given $\left\|\boldsymbol{T}_{m}\right\| \leq \delta_{2}$, where $\delta_{2}>0$ is an unknown and bounded constant.

Defined $\tilde{\delta}_{2}=\hat{\delta}_{2}-\delta_{2}$, which is estimate error of $\delta_{2}$, and $\hat{\delta}_{2}$ is estimate value of $\delta_{2}$. Similarly to the attitude sub-system, the control law can be designed as follows:

$$
\begin{aligned}
\boldsymbol{u}(t)=\boldsymbol{K}_{p} \boldsymbol{J} \tilde{\boldsymbol{\omega}}+\boldsymbol{\omega}^{\times} \boldsymbol{J} \boldsymbol{\omega}_{d}+\frac{c_{2} \hat{\delta}_{2}}{\|\boldsymbol{J} \tilde{\boldsymbol{\omega}}\|+b_{2}} \boldsymbol{J} \tilde{\boldsymbol{\omega}} \\
\dot{\hat{\delta}}_{2}=\frac{a_{2} c_{2}}{\|\boldsymbol{J} \tilde{\boldsymbol{\omega}}\|+b_{2}} \tilde{\boldsymbol{\omega}}^{T} \boldsymbol{J}^{T} \boldsymbol{J} \tilde{\boldsymbol{\omega}}
\end{aligned}
$$

Where $a_{2}, b_{2}>0, c_{2}>1$ are constants to be designed. Define Lyapunov function $V_{3}=V_{2}+1 /\left(2 a_{2}\right) \tilde{\delta}_{2}^{2}$, Similarly to the attitude subsystem, one has

$$
\dot{V}_{3} \leq-\tilde{\boldsymbol{\omega}}^{\mathrm{T}} \boldsymbol{J}^{\mathrm{T}} \boldsymbol{K}_{p} \boldsymbol{J} \tilde{\boldsymbol{\omega}}+\|\boldsymbol{J} \tilde{\boldsymbol{\omega}}\| \delta_{2}-\frac{c_{2} \delta_{2}\|\boldsymbol{J} \tilde{\boldsymbol{\omega}}\|^{2}}{\|\boldsymbol{J} \tilde{\boldsymbol{\omega}}\|+b_{2}}
$$

\subsection{Main Result}

Theorem: Consider the spacecraft system (1) and (2), if the control laws are given by (8) and (19), the adaptive estimators are defined by (9) and (20), and the conditions satisfies $\|\tilde{\boldsymbol{q}}\|>b_{1} /\left(c_{1}-1\right)$, $\|\tilde{\boldsymbol{\omega}}\|>b_{2} /\left(\|\boldsymbol{J}\|\left(c_{2}-1\right)\right)$, then close-loop system is stable, moreover, the attitude of spacecraft could converge to a small neighborhood of the desired attitude.

proof: If $\|\tilde{\boldsymbol{q}}\| \delta_{1}-c_{1} \delta_{1}\|\tilde{\boldsymbol{q}}\|^{2} /\left(\|\tilde{\boldsymbol{q}}\|+b_{1}\right) \leq 0$, that is $\|\tilde{\boldsymbol{q}}\|>b_{1} /\left(c_{1}-1\right)$, then equation (10) is transformed into

$$
\dot{V}_{1} \leq-\tilde{\boldsymbol{q}}^{\mathrm{T}} \boldsymbol{K}_{d} \tilde{\boldsymbol{q}}
$$

Similarly, if $\|\tilde{\boldsymbol{\omega}}\|>b_{2} /\left(\|\boldsymbol{J}\|\left(c_{2}-1\right)\right)$, then equation (21) is rewritten into

$$
\dot{V}_{3} \leq-\tilde{\boldsymbol{\omega}}^{\mathrm{T}} \boldsymbol{J}^{\mathrm{T}} \boldsymbol{K}_{p} \boldsymbol{J} \tilde{\boldsymbol{\omega}}
$$

From equation (22) and (23), if one chooses Lyapunov function $V=V_{1}+V_{3}$, the derivative of $V$ is $\dot{V}=\dot{V}_{1}+\dot{V}_{3} \leq 0$. Therefore, the close-loop system is stable.

From equation (22), the attitude tracking error $\tilde{\boldsymbol{q}}$ is ultimately bounded stable, and the stable region is $S_{\tilde{\boldsymbol{q}}}=\left\{\tilde{\boldsymbol{q}}\|\tilde{\boldsymbol{q}}\| \leq b_{1} /\left(c_{1}-1\right)\right\}$. From equation (23), the angular velocity tracking error $\tilde{\boldsymbol{\omega}}$ is also ultimately bounded stable, and the stable region is $S_{\tilde{\omega}}=\left\{\tilde{\boldsymbol{\omega}}\|\tilde{\boldsymbol{\omega}}\| \leq b_{2} /\left(\|\boldsymbol{J}\|\left(c_{2}-1\right)\right)\right\}$. Therefore, if one can choose appropriate parameters such that $b_{1}>0, c_{1}>1$ and $b_{2}>0, c_{2}>1$, then, the designed control law can guarantee that the attitude of spacecraft could converge to a small neighborhood of the desired attitude. 


\section{Simulation}

In simulation, the spacecraft inertia matrix $\boldsymbol{J}$, attitude rotation matrix $\boldsymbol{B}$ and disturbance $\boldsymbol{d}, \boldsymbol{f}$ are

$$
\begin{aligned}
\boldsymbol{J} & =\left[\begin{array}{ccc}
1320 & 194 & 176 \\
194 & 9600 & 145 \\
176 & 145 & 6400
\end{array}\right]\left(\mathrm{kg} \cdot \mathrm{m}^{2}\right), \quad \boldsymbol{B}=\frac{1}{\cos \psi}\left[\begin{array}{ccc}
\cos \theta & 0 & \sin \theta \\
\cos \theta \sin \psi & 1 & \sin \theta \sin \psi \\
-\sin \theta & 0 & \cos \theta
\end{array}\right] \\
\boldsymbol{f} & =\left[\begin{array}{c}
0.05+0.1 \sin t \\
0.01+0.03 \sin 2 t \\
0.01+0.02 \sin 3 t
\end{array}\right](\mathrm{rad} / \mathrm{s}), \quad \boldsymbol{d}=\left[\begin{array}{c}
0.1+0.1 \sin 5 t \\
0.2+0.15 \cos (2 t+\pi / 6) \\
0.3+0.15 \cos (2 t+\pi / 4)
\end{array}\right] \times 10^{-2}(\mathrm{~N} \cdot \mathrm{m})
\end{aligned}
$$

The aim is control spacecraft attitude from initial attitude $\boldsymbol{q}_{0}=\left[\begin{array}{lll}12.6^{\circ} & -5^{\circ} & -27.29^{\circ}\end{array}\right]^{\mathrm{T}}$ to the terminal attitude $\boldsymbol{q}_{d}=\boldsymbol{0}$ along the desired attitude trajectory $\boldsymbol{q}_{r}=\boldsymbol{q}_{0}+\left(\frac{10 t^{3}}{20^{3}}-\frac{15 t^{4}}{20^{4}}+\frac{6 t^{5}}{20^{5}}\right)\left(\boldsymbol{q}_{d}-\boldsymbol{q}_{0}\right)$. To this end, we assign the gain matrix $\boldsymbol{K}_{d}=10 \boldsymbol{I}_{3}, \boldsymbol{K}_{p}=40 \boldsymbol{I}_{3}$. Moreover, the initial values of the estimator are set to $\hat{\delta}_{1}(0)=\hat{\delta}_{2}(0)=0$, and adaptive parameters are $b_{1}=b_{2}=0.005$. Other parameters are given by $a_{1}=1$, $a_{2}=0.01$.

For attitude error, two cases are considered about the different adaptive parameter $c_{1}$ under the same parameter $c_{2}=2$, respectively. One is $c_{1}=3$, then, one can calculate the stable region of $\tilde{\boldsymbol{q}}$ is $S_{\tilde{\boldsymbol{q}}}=\left\{\tilde{\boldsymbol{q}}\|\tilde{\boldsymbol{q}}\| \leq 0.143^{\circ}\right\}$. The other one is $c_{1}=4$, then, one can calculate the stable region of $\tilde{\boldsymbol{q}}$ is $S_{\tilde{\boldsymbol{q}}}=\left\{\tilde{\boldsymbol{q}}\|\tilde{\boldsymbol{q}}\| \leq 0.095^{\circ}\right\}$.

The simulation results are displayed in Fig.1 and Fig.2.

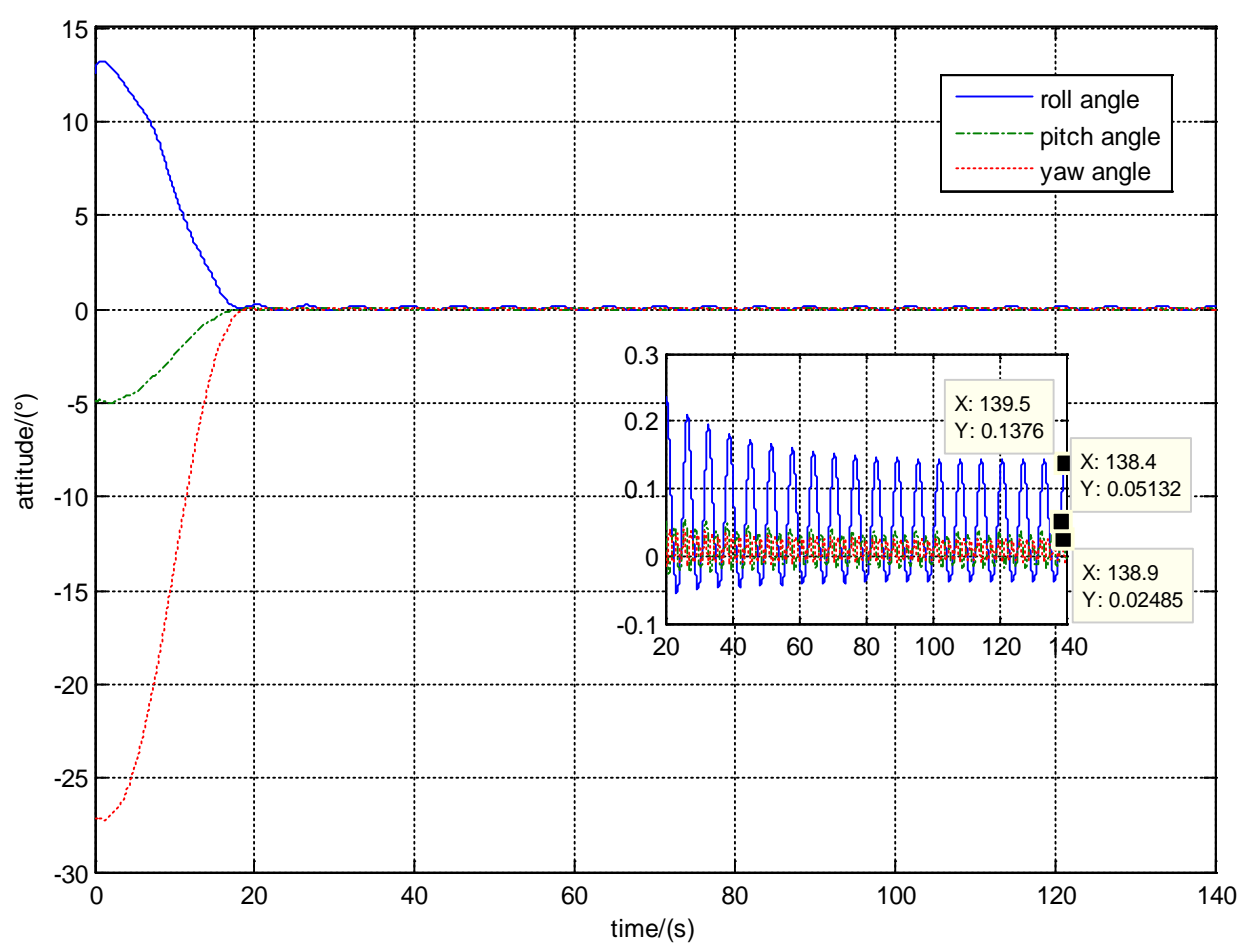

Fig.1. Attitude curve with $c_{1}=3$ 


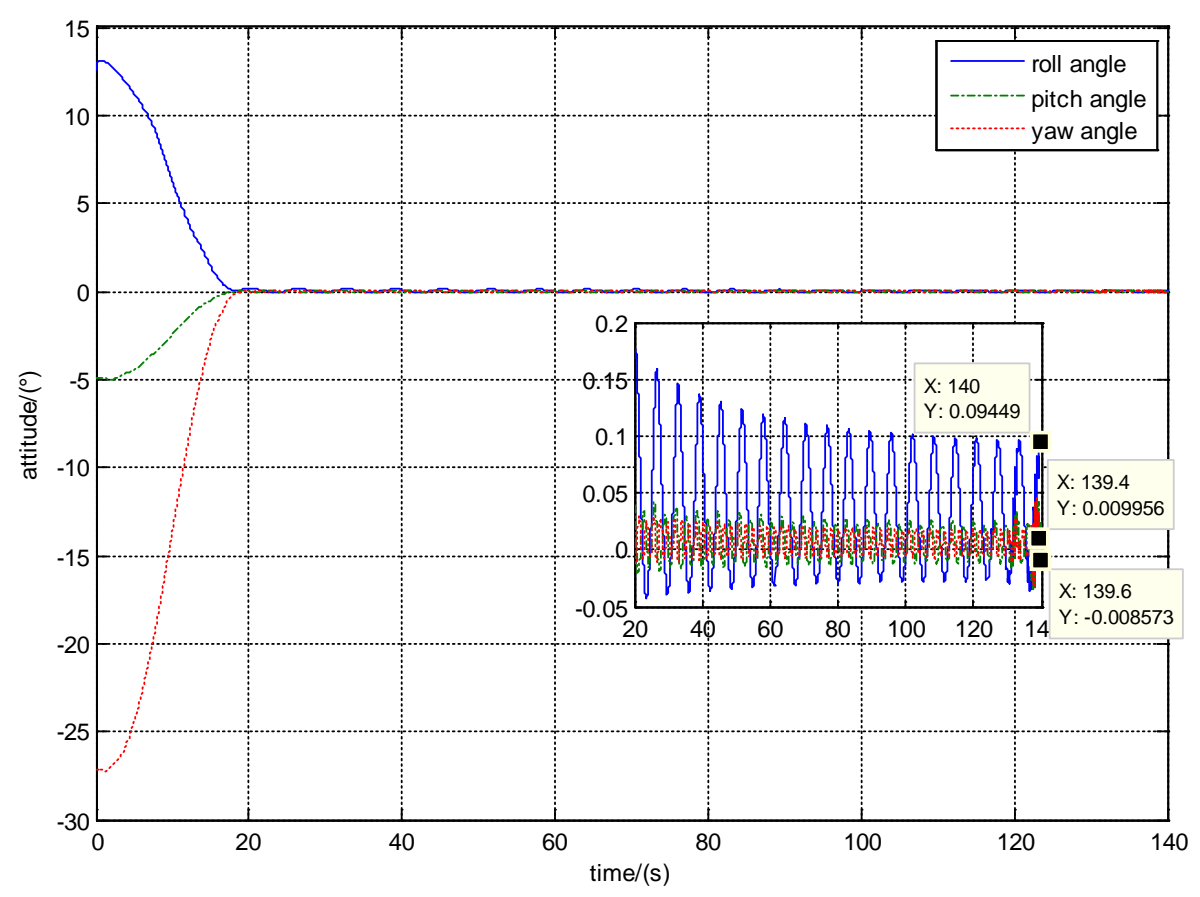

Fig.2. Attitude curve with $c_{1}=4$

It can be seen from the simulation results that the attitude of the closed-loop system would take about 140s to arrive at the stable region correspondingly with the proposed control. Since there are mismatch external disturbances in spacecraft model, the attitude of spacecraft would converge into a small neighborhood of the reference attitude, and the control precision is better.

\section{Conclusion}

An attitude control method is proposed for MIMO spacecraft subject to external disturbances in the paper. In control design, the disturbance is fully considered and resolved by virtual control design of subsystem. On the other hand, the nonlinear of model is utilized adequately, which make the designed controller add to spacecraft dynamics equation and receive a nonlinear close-loop error equation. The reference worth of proposed controller is higher in engineering study.

\section{References}

[1] L. Wang, X. D. Liu, Y. Z. Sheng, et al, Disturbance Observer Based Exponential Time-varying Sliding Mode for Re-entry Attitude Control, Chinese Space Science and Technology, 8(2013) 31-38.

[2] Z. Y. Chu, J. Cui and F. C. Sun, Fuzzy Adaptive Disturbance- Observer -Based Robust Tracking Control of Electrically Driven Free-Floating Space Manipulator, IEEE Systems Journal, 8(2014) 343-352.

[3] H. Y. Chai, Y. P. Yan and L. Guo, Feedback Linearization Design for Permanent Magnet Synchronous Motor with Disturbance Observer, Proceedings of the 32nd Chinese Control Conference,(2013) 2739-2744,

[4] H. Lu, N. Wang, J. Z. Qiao, et al, Nonlinear Disturbance Observer Based Command Filtered Backstepping Control for Missile System, Proceedings of the 32nd Chinese Control Conference, (2013) 4311-4316.

[5] S. B. Wang, X. M. Wang, R. Xie, et.al, Robust Backstepping Control Based on Disturbance Observer for Hypersonic Vehicle, Control and Decision, 28(2013) 507-1512. 
[6] S. Jin, Z. X Zhang, S. H. Li, Design of Guidance Law Based on Non- singular Terminal Sliding Mode Control and Finite-Time Disturbance Observer, 13th IEEE Workshop on Variable Structure Systems, (2014).

[7] P. Li and G. H. Yang, A new adaptive control approach for nonlinear strict-feedback systems using nonlinearly parameterized fuzzy approximators, International Journal of Systems Science, 42 (2011) 517-527. 